Article

\title{
Quercitrin Stimulates Hair Growth with Enhanced Expression of Growth Factors via Activation of MAPK/CREB Signaling Pathway
}

\author{
Jaeyoon Kim ${ }^{1,2}{ }^{1}$, Soon Re Kim ${ }^{3}$, Yun-Ho Choi ${ }^{1}$, Jae young Shin ${ }^{1}$, Chang Deok Kim ${ }^{2}$, \\ Nae-Gyu Kang ${ }^{1}$, Byung Cheol Park ${ }^{3,4}$ and Sanghwa Lee ${ }^{1, *}$ \\ 1 LG Household \& Health Care (LG H\&H) R\&D Center, 70, Magokjoongang 10-ro, Gangseo-gu, \\ Seoul 07795, Korea; kjy5281@lghnh.com (J.K.); youknow@lghnh.com (Y.-H.C.); sjy2811@lghnh.com (J.y.S.); \\ ngkang@lghnh.com (N.-G.K.) \\ 2 Department of Dermatology, School of Medicine, Chungnam National University, 266, Munwha-ro, Jung-gu, \\ Deajeon 35015, Korea; cdkimd@cnu.ac.kr \\ 3 Basic and clinical Hair institute, Dankook University, 201, Manghyang-ro, Dongnam-gu, Cheonan-si, \\ Chungcheongnam-do 31116, Korea; s1715@nate.com (S.R.K.); 4exodus@dankook.ac.kr (B.C.P.) \\ 4 Department of Dermatology, Dankook University Hospital, 201, Manghyang-ro, Dongnam-gu, Cheonan-si, \\ Chungcheongnam-do 31116, Korea \\ * Correspondence: shleek@lghnh.com; Tel.: +82-2-6980-1210
}

Academic Editors: George Grant and Derek J. McPhee

Received: 18 August 2020; Accepted: 1 September 2020; Published: 2 September 2020

\begin{abstract}
The present study aimed to investigate the molecular mechanism of quercitrin, a major constituent of Hottuynia cordata extract, for its hair growth stimulating activities in cultured human dermal papilla cells (hDPCs). Quercitrin enhanced the cell viability and cellular energy metabolism in cultured hDPCs by stimulating the production of $\mathrm{NAD}(\mathrm{P}) \mathrm{H}$ and mitochondrial membrane potential $(\Delta \Psi)$. The expression of Bcl2, an essential marker for anagen hair follicle and cell survival, was increased by quercitrin treatment. Quercitrin also increased the cell proliferation marker Ki67. The expression of growth factors-such as bFGF, KGF, PDGF-AA, and VEGF-were increased by quercitrin both in mRNA and protein levels. In addition, quercitrin was found to increase the phosphorylation of Akt, Erk, and CREB in cultured hDPCs, while inhibitors of MAPKs reversed the effects of quercitrin. Finally, quercitrin stimulated hair shaft growth in cultured human hair follicles. Our data obtained from present study are in line with those previously reported and demonstrate that quercitrin is (one of) the active compound(s) of Hottuynia cordata extract which showed hair growth promoting effects. It is strongly suggested that the hair growth stimulating activity of quercitrin was exerted by enhancing the cellular energy metabolism, increasing the production of growth factors via activation of MAPK/CREB signaling pathway.
\end{abstract}

Keywords: quercitrin; human DPCs; hair growth; growth factors; MAPK; CREB

\section{Introduction}

Androgenetic alopecia (AGA) is the most common type of hair loss in men and is a naturally progressive disorder [1]. In the process of AGA, large terminal scalp hairs are gradually replaced by thinner and smaller vellus hairs, which is called hair follicle miniaturization. The pathogenesis and underlying molecular mechanisms of AGA are not yet fully understood but androgen hormone, dihydrotestosterone (DHT) is suspected to be the most potent causative agent in genetically predisposed individuals [2].

Although AGA is not a life-threatening disease it has great psychosocial impact on affected patients, resulting in a significant impairment of quality of life [3]. Despite tremendous efforts for 
curing AGA, just two drugs-minoxidil and finasteride-were approved by the US Food and Drug Administration (FDA). Among those, minoxidil is the only topical treatment for AGA but the side effects such as pruritus, dermatitis, and irritation have been reported [4].

The hair follicle undergoes three cyclic stages of growth; anagen (proliferation), catagen (involution), and telogen (resting) [5]. Dermal papilla cells (DPCs), differentiated from mesenchymal stem cells and located in the core of hair follicle, are one of the major regulators of hair cycle which controls external stimuli and signals delivered through cytokines and junctions [6]. In AGA, repeated hair cycles with shortened anagen result in hair follicle miniaturization, characterized by depletion of anagen follicles and presence of vellus hairs on affected scalp [7]. Mesenchymal stem cell-derived signals and growth factors from DPCs could influence hair growth through proliferation of hair follicle cells. They could prolong the anagen (KGF), stimulate hair follicle development ( $\beta$-catenin), or suppress apoptotic cues (Bcl-2) [8]. Stimulating the production of growth factors from DPCs such as bFGF, KGF, PDGF-AA, and VEGF could be effective therapeutic candidates for activating hair cycle and growth.

We have previously reported that the extract of Houttuynia cordata (HCE) promoted hair growth in cultured hDPCs and human hair follicle culture model, conferred by elongation of hair follicle anagen [9]. The HCE may contain thousands of chemicals but we have found the presence of four compounds-chlorogenic acid, rutin, quercitrin, and quercetin-as major constituents. Among these, quercitrin was the most abundant one with concentration of approximately $16 \mathrm{mg} / \mathrm{mL}(1.6 \%)$. We have postulated that most (if not all) of the hair growth promoting activity of HCE had come from quercitrin.

Quercitrin (quercetin-3-O-rhamnoside) is a natural flavonoid widely found in the flowers, leaves, and fruits of various plants and was reported as anti-inflammatory [10] and anti-oxidant agents [11]. However, the effects of quercitrin on hair growth and alopecia treatment, as we know, were not reported.

In this study, physiological effects of quercitrin on cultured human DPCs and its molecular mechanisms were investigated to prove our hypothesis in which quercitrin is the active ingredient of HCE in stimulation of hair growth.

As expected, quercitrin was found to stimulate the mitochondrial energy metabolism and enhance proliferative capacity, accompanied by increased production of growth factors like bFGF, KGF, PDGF-AA, and VEGF, essential for hair growth. Furthermore, the expression of Bcl2 and Ki67, typical markers for cell survival and anagen extension, was also increased. The cell signal transduction elements-such as Akt, Erk, CREB and several receptor tyrosine kinases-were found to be stimulated by quercitrin. In addition, quercitrin enhanced the hair shaft growth in cultured human hair follicles. Our data strongly suggest that the hair growth stimulating activity of HCE comes mainly from quercitrin and that quercitrin possesses possible therapeutic potential for preventing and/or treating the hair loss.

\section{Results}

\subsection{Quercitrin Increased Cell Viability in Cultured Human DPCS}

The treatment of quercitrin increased the viability of DPCs in a concentration dependent manner. $\mathrm{NAD}(\mathrm{P}) \mathrm{H}$ generation, presented as cell viability, was increased by $9.6 \%, 24.4 \%$, and $39.0 \%$ in the presence of $0.1,1$, and $10 \mathrm{nM}$ quercitrin, respectively. Above $10 \mathrm{nM}$ concentrations, the cell viability was reached to a plateau (Figure 1b). The increments were comparable with that of $100 \mathrm{nM}$ of minoxidil, approved topical hair growth stimulating medicine, a positive control.

Because $\mathrm{NAD}(\mathrm{P}) \mathrm{H}$ production was markedly increased by quercitrin treatment, the changes in mitochondrial membrane potential $(\Delta \Psi)$ were investigated. The mitochondrial membrane potential indicates the capacity for energy generation in mitochondria. As shown in Figure 2, the membrane potential was increased in a concentration dependent manner. The treatment of quercitrin at concentration of 10 and $100 \mathrm{nM}$ significantly increased the membrane potential by $34.0 \%$ and $63.2 \%$, respectively. Combining the data in Figures 1 and 2, the treatment of quercitrin resulted in potentiation of energy metabolism in cultured hDPCs. 
<smiles>C[C@@H]1O[C@H](Oc2c(-c3ccc(O)c(O)c3)oc3cc(O)cc(O)c3c2=O)[C@H](O)[C@@H](O)[C@@H]1O</smiles>

(a)

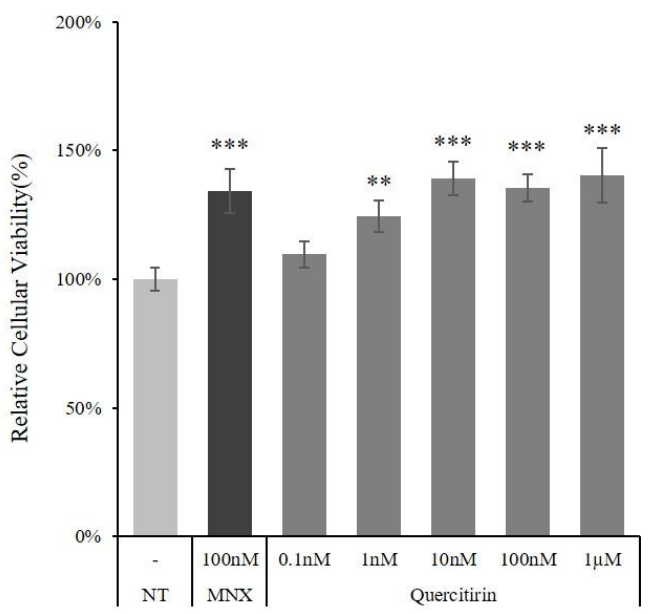

(b)

Figure 1. Quercitrin enhanced cell viability in cultured hDPCs. (a) Quercitrin chemical structure. (b) Cell viability was assessed using CCK-8 assay kit after quercitrin treatment (0.1, 1, 10, $100 \mathrm{nM}$ and $1 \mu \mathrm{M})$ for $24 \mathrm{~h}$. The value of non-treated control was taken to be $100 \%$. N.T, non-treated control; MNX, minoxidil. Significantly different compared with N.T $\left({ }^{*} p<0.05,{ }^{* *} p<0.01,{ }^{* * *} p<0.001\right)$.

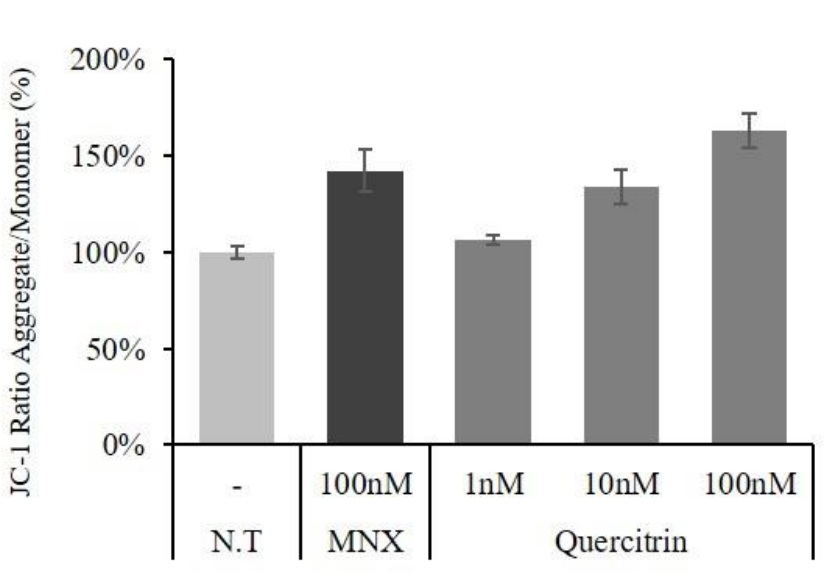

(a)

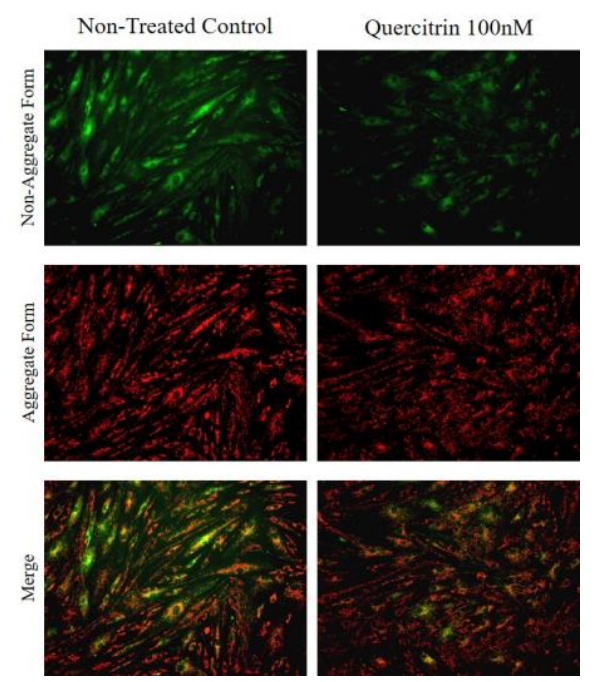

(b)

Figure 2. Effect of quercitrin on cellular energy metabolism in cultured hDPCs. (a) Mitochondrial membrane potential was measured after quercitrin treatment $(1,10$, and $100 \mathrm{nM})$ for $24 \mathrm{~h}$. The $100 \mathrm{nM}$ minoxidil was used as a positive control. (b) JC-1 monomer form was seen as green and aggregate form as red by fluorescent microscopy. N.T, non-treated control; MNX, minoxidil. Significantly different compared with N.T $\left({ }^{*} p<0.05,{ }^{* *} p<0.01,{ }^{* * *} p<0.001\right)$.

\subsection{Quercitrin Enhanced Bcl2 Expression}

The mRNA expression level of cell viability related genes was assessed in cultured hDPCs. The expression of Bad, an apoptotic marker in mitochondria, was significantly decreased by quercitrin treatment. On the contrary, an anti-apoptotic gene $\mathrm{Bcl} 2$ level was significantly increased by $47 \%$ and 54\% with 10 and $100 \mathrm{nM}$ quercitrin, respectively. In addition, cell proliferation marker, Ki67 was also increased by quercitrin in a concentration dependent manner (Figure 3a). Western blot analysis revealed that quercitrin also significantly increased the amount of $\mathrm{Bcl} 2$ protein by $66.2 \%$ with $100 \mathrm{nM}$ treatment (Figure 3b,c). 


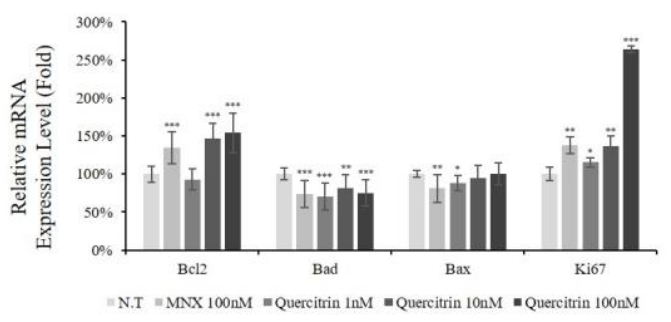

(a)

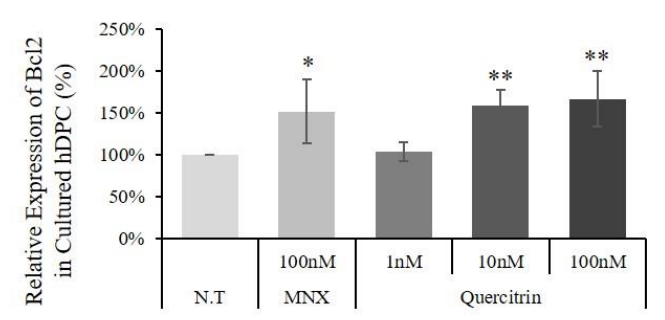

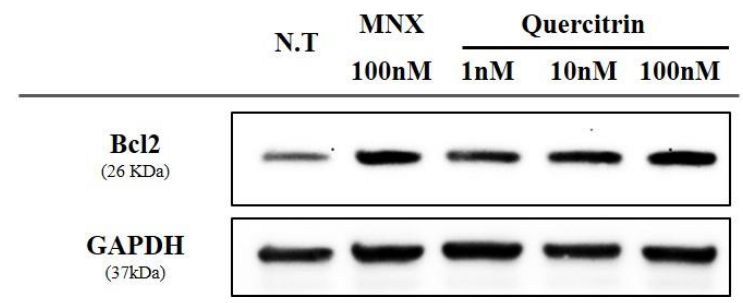

(b)

(c)

Figure 3. Effect of quercitrin on mRNA expression levels of proliferative/apoptotic genes and protein level of Bcl2 in cultured hDPCs. The cultured DPCs were harvested after quercitrin treatment $(1,10$, and $100 \mathrm{nM}$ ) for $24 \mathrm{~h}$. (a) The mRNA expression levels of Bcl2, Bad, Bax and Ki67 genes in cultured hDPCs were measured by real-time PCR. (b) Whole cell lysates (50 $\mu \mathrm{g}$ protein) from DPCs were analyzed by immunoblotting to determine the levels of $\mathrm{Bcl} 2$, and (c) the band intensity was quantitated. N.T, non-treated control; MNX, minoxidil. Significantly different compared with N.T $\left({ }^{*} p<0.05\right.$, ** $\left.p<0.01,{ }^{* * *} p<0.001\right)$.

\subsection{Quercitrin Increased mRNA Expression of Growth Factors and Their Receptors}

The DPCs secrete myriad of growth factors to regulate hair follicle growth. To specify the factors affecting metabolic stimulation and cell viability, the mRNA expression of growth factors and growth factor receptors was evaluated. The expression levels of 19 growth factor genes and 15 growth factor receptor genes were measured. Among 34 genes tested, the expression of 8 genes (bFGF, KGF, IGFBP2, TGFb1, VEGFA, FGFR1, PDGFRa, and PDGFRb) was increased by quercitrin treatment. Among growth factors, mRNA expression of bFGF, KGF and VEGFA was significantly increased by 50.1\%, 79.5\%, and $32.7 \%$ at $100 \mathrm{nM}$ quercitrin treatment, respectively. For growth factor receptors, the expression level of FGFR1 and PDGFRa was significantly increased (Figure 4).

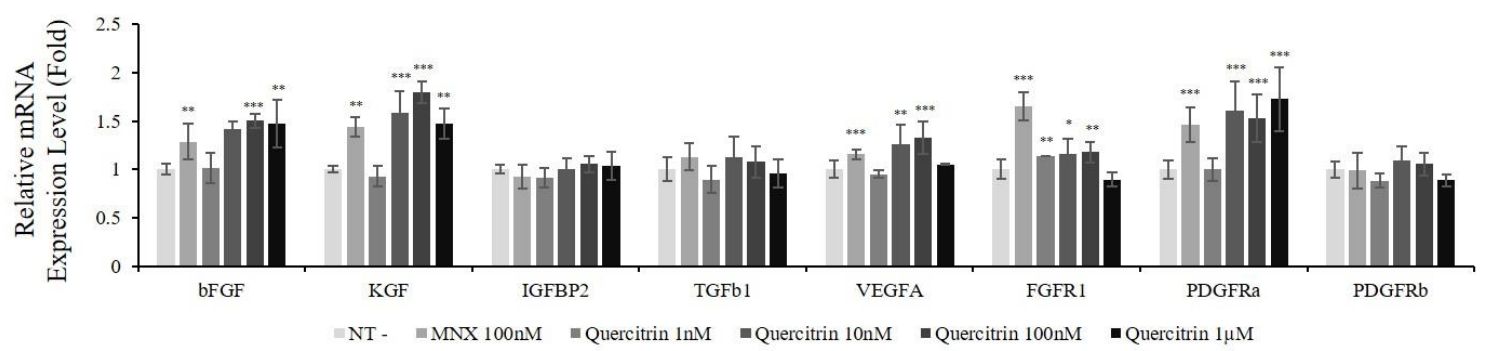

Figure 4. Effect of quercitrin on mRNA expression levels of growth factor genes in cultured hDPCs. The cells were harvested after quercitrin treatment $(1,10,100 \mathrm{nM}$ and $1 \mu \mathrm{M})$ for $24 \mathrm{~h}$. The mRNA expression levels of eight genes in cultured hDPCs were measured by real-time PCR. N.T, non-treated control; MNX, minoxidil. Significantly different compared with N.T $\left({ }^{*} p<0.05,{ }^{* *} p<0.01,{ }^{* * *} p<0.001\right)$. 


\subsection{Quercitrin Increased the Protein Expression of Growth Factors}

To elucidate whether quercitrin affect the gene expression of growth factors and their receptors to protein level, dot blot analysis was performed. The amount of bFGF, KGF, PDGF-AA, and VEGF, which were reported to enhance the cell viability of DPCs [12], was significantly increased by quercitrin treatment (Figure 5).

(a)

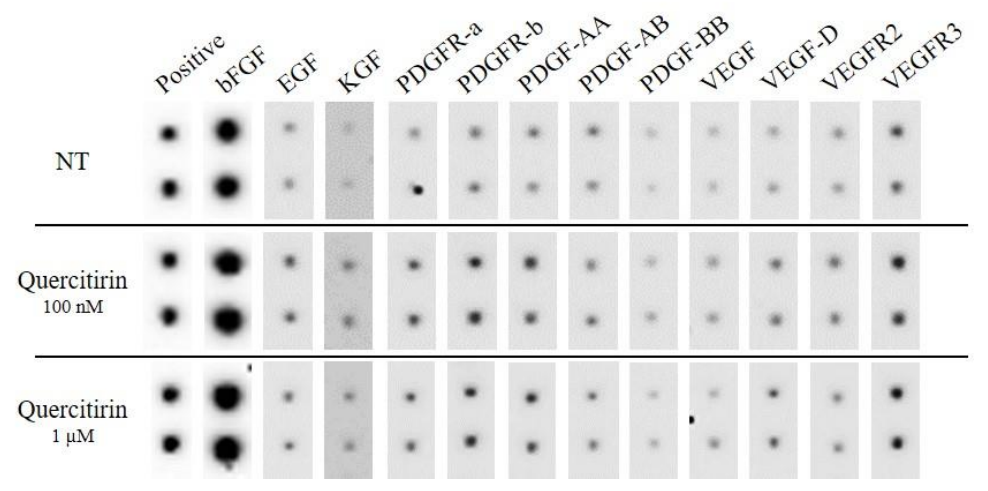

(b)

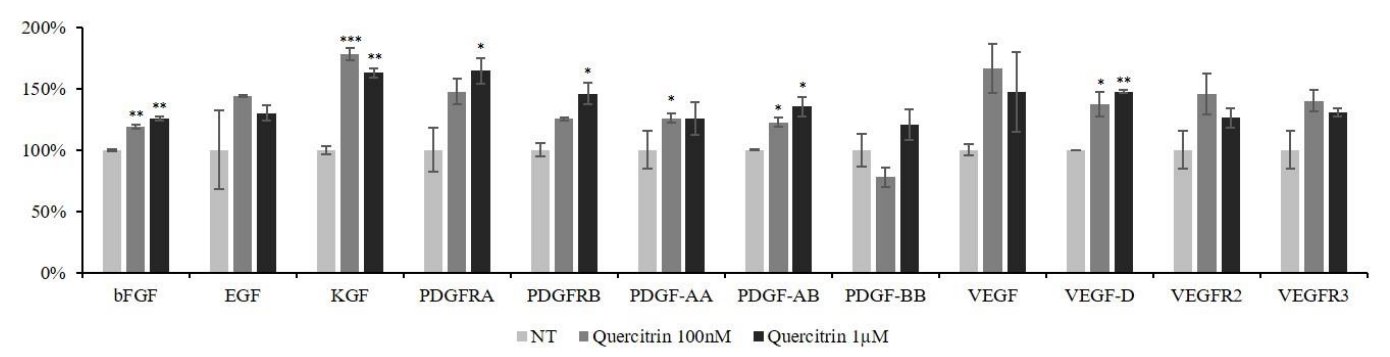

Figure 5. Effect of quercitrin on the expression of growth factors in cultured hDPCs. The DPCs were treated with quercitrin $(0,100 \mathrm{nM}$ and $1 \mu \mathrm{M})$ for $24 \mathrm{~h}$, and then collected. Cells cultured with vehicle medium were used as non-treated control. Total of 41 types of human growth factors were analyzed. (a) The 12 types of growth factors and receptors were displayed and (b) the band intensity was quantitated. N.T, non-treated control. Positive, biotin-conjugated IgG. Significantly different compared with N.T $\left({ }^{*} p<0.05,{ }^{* *} p<0.01,{ }^{* * *} p<0.001\right)$.

Protein levels of bFGF and KGF both in culture medium and cell lysate were further investigated using ELISA to differentially assess the intracellular and secreted form. The intracellular bFGF and KGF were significantly increased by quercitrin treatment (Figure 6a,c). Furthermore, quercitrin at concentration of $100 \mathrm{nM}$ also increased the secretion of bFGF and KGF by $36.5 \%$ and $27.3 \%$, respectively (Figure $6 \mathrm{~b}, \mathrm{~d}$ ). Taken together, quercitrin enhanced not only the production of bFGF and KGF but also the secretion of them.

\subsection{Quercitrin Induced Phosphorylation of Akt, Erk, and CREB}

To clarify the molecular action mechanism of quercitrin, the MAP kinase molecules, which play pivotal roles in cellular signal cascades, were investigated. Among phosphorylated MAP kinase proteins, phospho-Akt and phospho-Erk were significantly increased. The protein levels of total Akt and Erk, on the other hand, were not changed. It was revealed that the phosphorylation of Akt and Erk tended to have a time dependency with maximum increases at a certain time point (Figure 7a). The ratio of pAkt/Akt was increased by $73.3 \%$ and $63.8 \%$, when $100 \mathrm{nM}$ of quercitrin was treated for 1 and $2 \mathrm{~min}$, respectively. On the other hand, pErk/Erk ratio was more prominently increased by $204.0 \%$ and $285.9 \%$, for 2 and 5 min of quercitrin treatment, respectively (Figure $7 \mathrm{~b}$ ). As a downstream target of Thr202-Erk phosphorylation, CREB phosphorylation at Ser133 site was examined [13]. The phospho 
(Ser133)-CREB/CREB level was significantly elevated by $671.4 \%, 680.0 \%$, and 244.0\%, when $100 \mathrm{nM}$ of quercitrin was treated for 5, 10, and $20 \mathrm{~min}$, respectively (Figure 7b).

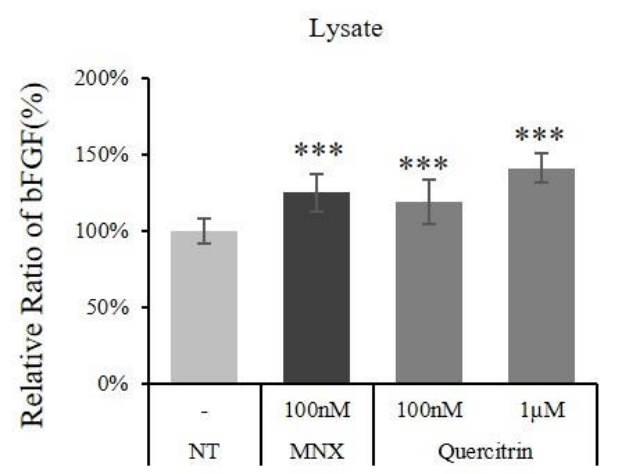

(a)

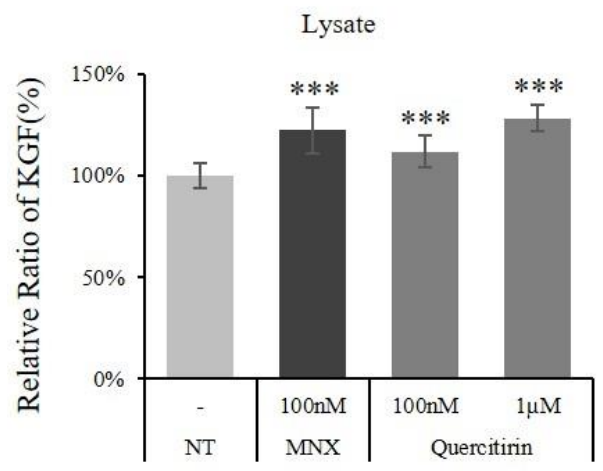

(c)

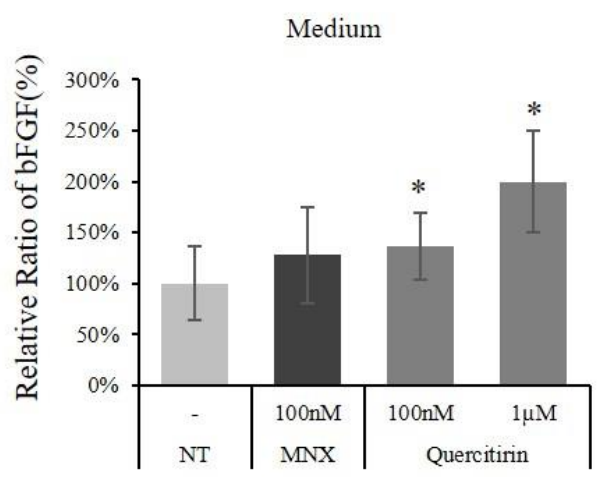

(b)

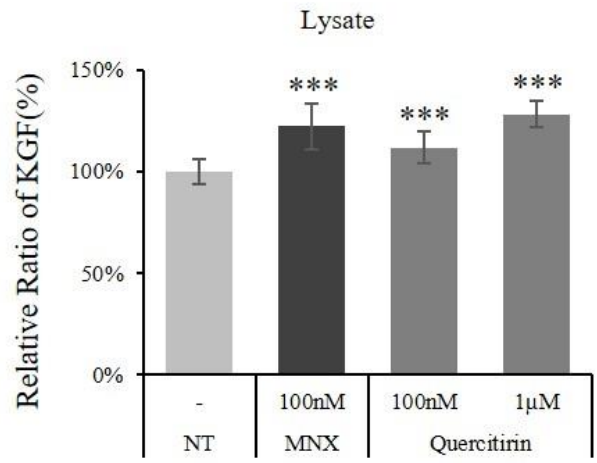

(d)

Figure 6. Effect of quercitrin on the protein expression of bFGF and KGF in cultured hDPCs. The DPCs were treated with quercitrin at concentrations of $1,10,100 \mathrm{nM}$ and $1 \mu \mathrm{M}$ for $24 \mathrm{~h}$. Whole cell lysates $(\mathbf{a}, \mathbf{c})$ and culture medium $(\mathbf{b}, \mathbf{d})$ of each cultured DPCs were analyzed by ELISA to determine the levels of bFGF $(a, b)$ and KGF $(c, d)$. N.T, non-treated control. Significantly different compared with N.T $\left({ }^{*} p<0.05,{ }^{* *} p<0.01,{ }^{* * *} p<0.001\right)$.

We scrutinized whether the inhibitors of MAP kinases could inhibit the quercitrin induced gene expressions to find out the relationship between enhanced gene expression and MAPKs. As shown in Figure 8, Erk inhibitor U0126 $(20 \mu \mathrm{M})$ significantly diminished the quercitrin stimulated expression of bFGF, KGF and Bcl2 while Akt inhibitor API-2 did not. The quercitrin-induced Ki67 expression, on the other hand, was inhibited by both U0125 and API-2. Our data suggest that quercitrin-stimulated expression of growth factors (bFGF and KGF) and Bcl2 was mediated by Erk activity and that both Erk and Akt were involved in enhanced cell proliferative potential by quercitrin. 
(a)

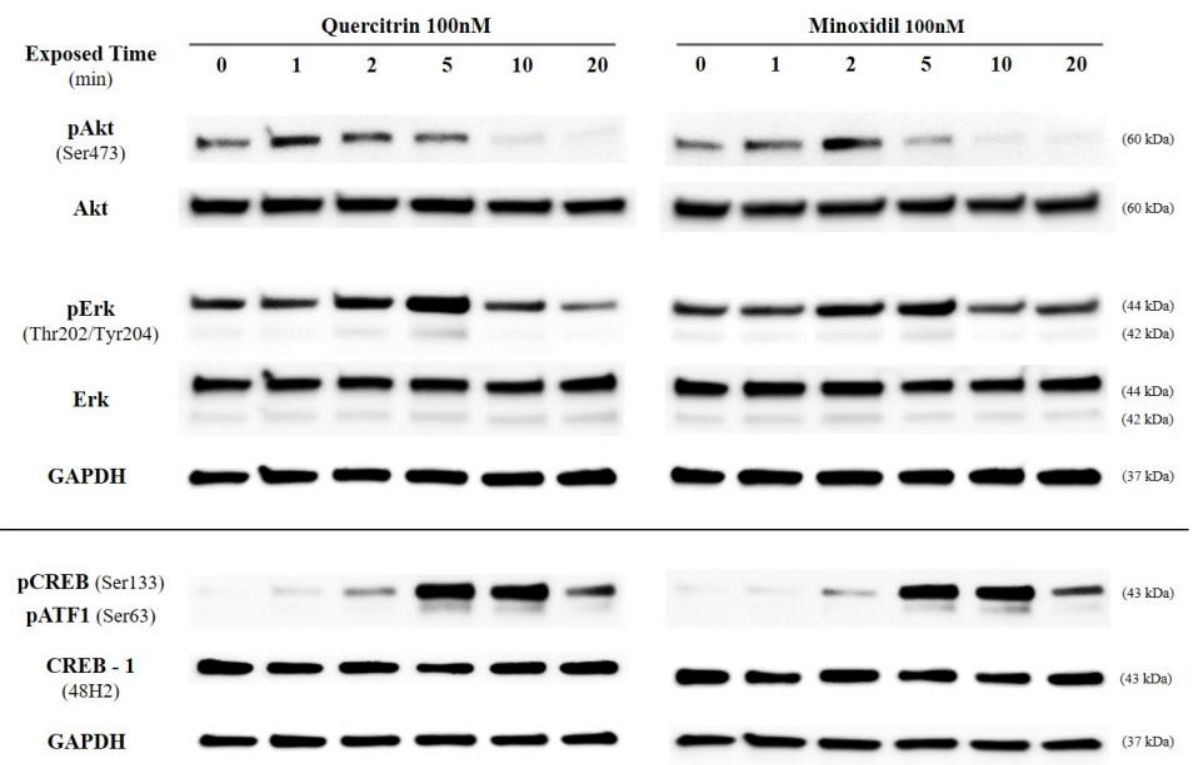

(b)
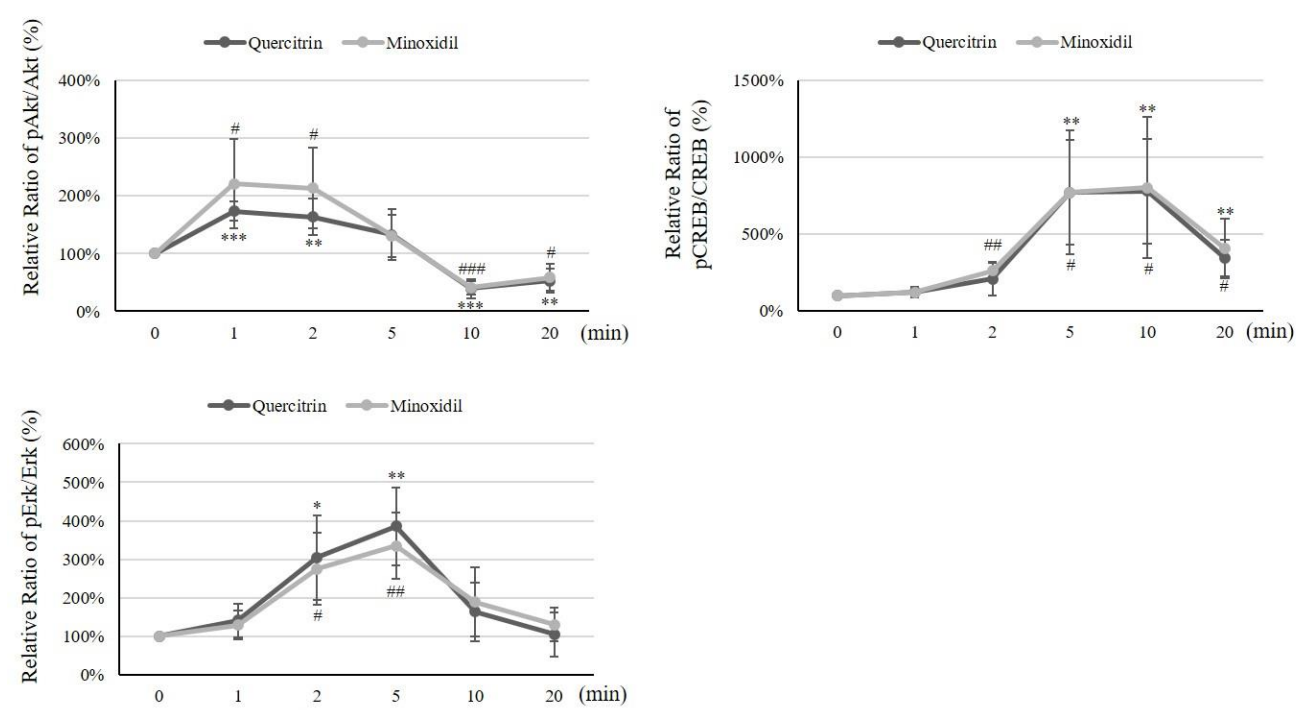

Figure 7. Effect of quercitrin on Akt, Erk, and CREB phosphorylation in cultured hDPCs. Quercitrin was treated for different times $(0,1,2,5,10$, and $20 \mathrm{~min})$. (a) Whole cell lysates were analyzed by immunoblotting to determine the levels of Akt, phospho-Akt, Erk, phospho-Erk, CREB and phospho-CREB. As an internal control, GAPDH were used. (b) The ratio of pAkt/Akt, pErk/Erk, and pCREB/CREB was calculated. N.T, non-treated control. The data represent the means of five independent samples. Significantly different compared with N.T (Quercitrin ${ }^{*} p<0.05,{ }^{* *} p<0.01$, *** $p<0.001$; Minoxidil \# $p<0.05$, \#\# $p<0.01$, \#\#\# $p<0.001$ ).

\subsection{Quercitrin Activated Receptor Tyrosine Kinases and Non-Receptor Tyrosine Kinases}

The receptor tyrosine kinase and its receptor activation pathways were well proved for the activation of Akt and Erk/MAPK pathways [14]. Therefore, we investigated the phosphorylation of tyrosine kinases using RTK dot blotting in cultured DPCs treated with quercitrin for $30 \mathrm{~s}$ and $1 \mathrm{~min}$. Total of 71 phosphorylated proteins were examined and 18 proteins were detected with significant increase by quercitrin treatment $(p<0.05), 8$ receptor tyrosine kinases and 10 non-receptor tyrosine kinases including Src family proteins (Figure 9). 

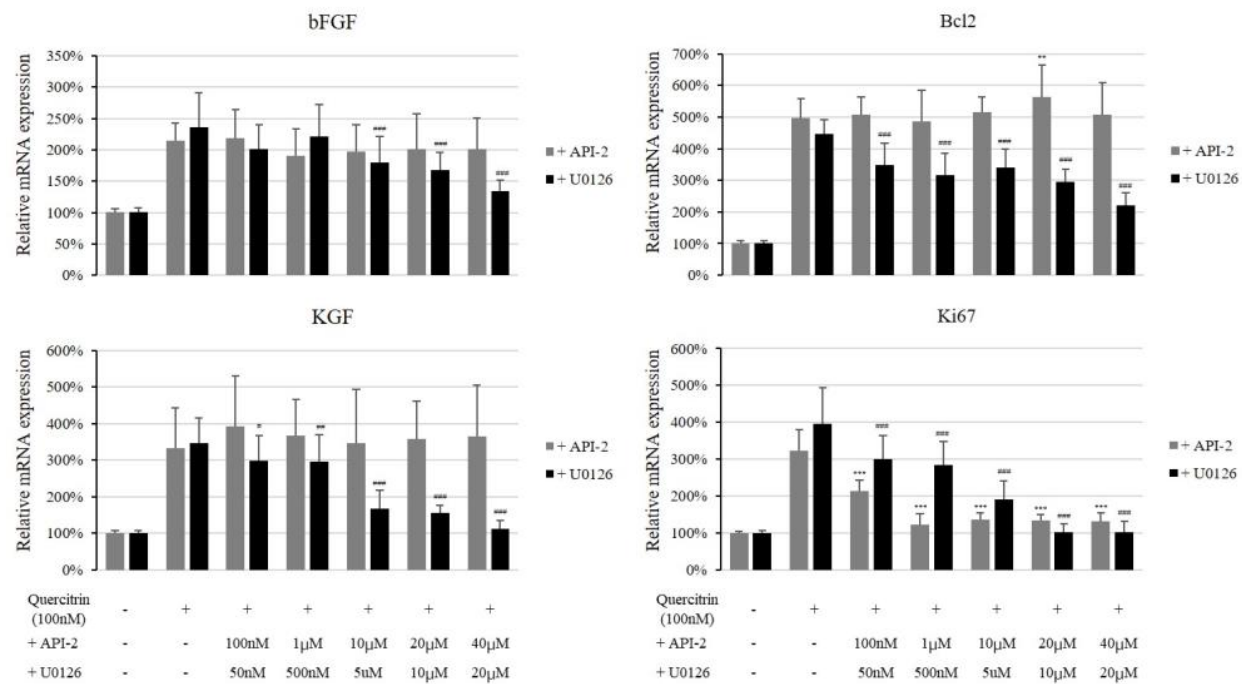

Figure 8. Effect of Akt inhibitor API-2 and Erk inhibitor U0126 on quercitrin stimulated gene expression. The cultured DPCs were harvested after $100 \mathrm{nM}$ quercitrin treatment with inhibitors (API-2 and U0125) for $24 \mathrm{~h}$. The mRNA expression levels of bFGF, KGF, Bcl2, and Ki67 were measured by real-time PCR. The data represent the means of six independent samples. Significantly different compared with $100 \mathrm{nM}$ quercitrin treatment (API-2 ${ }^{*} p<0.05,{ }^{* *} p<0.01,{ }^{* * *} p<0.001$; U0126 \# $p<0.05$, \#\# $p<0.01$, $\# \#$ \# $<0.001)$.

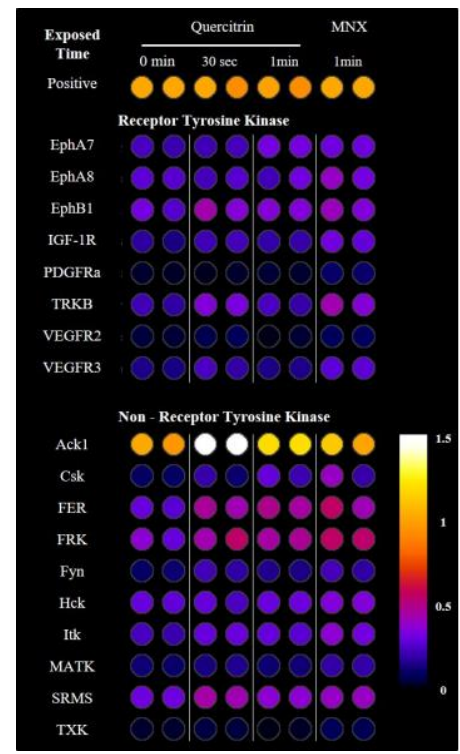

Figure 9. Quercitrin stimulated the receptor tyrosine kinases and non-receptor tyrosine kinases in cultured hDPCs. $100 \mathrm{nM}$ of quercitrin was treated for appropriate times $(0,0.5,1 \mathrm{~min})$. Whole cell lysates were analyzed by immunoblotting to determine the level of phospho-tyrosine following manufacturer's instruction. Total of 71 types of tyrosine kinase were analyzed. The 8 receptor tyrosine kinases and 10 non-receptors tyrosine kinases were displayed (Significantly different compared with $0 \mathrm{~min}, p<0.05$ ). Positive, biotin-conjugated IgG.

\subsection{Quercitrin Stimulated Hair Growth Ex Vivo}

The effect of quercitrin on human hair growth was investigated in human hair follicle organ culture model. Quercitrin significantly increased the hair growth compared with non-treated control (Figure 10). On day 4 of culture, quercitrin increased the length of hair shaft by $62.4 \%(5 \mu \mathrm{M})$ and $70.0 \%$ $(10 \mu \mathrm{M})$ compared with non-treated control. A positive control minoxidil showed a comparable result. 
(a)

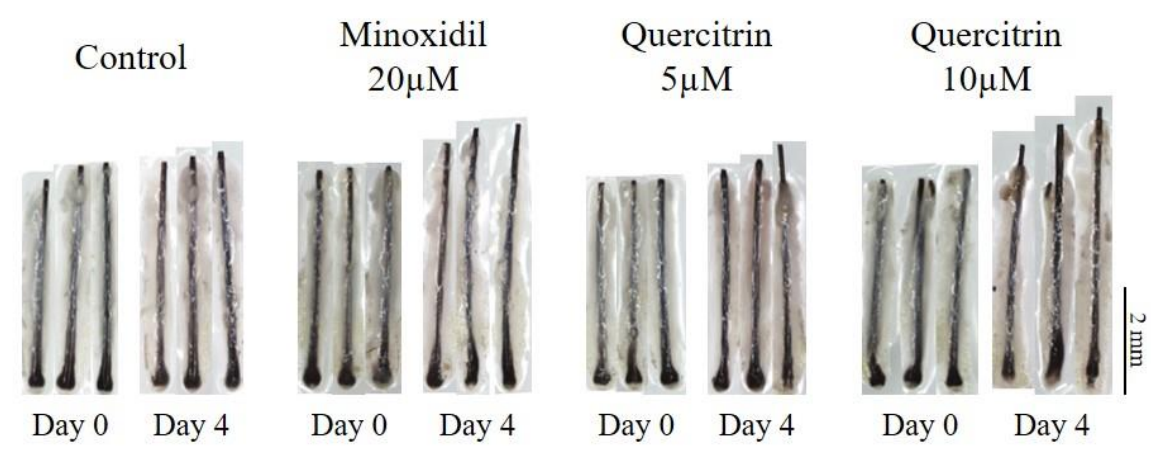

(b)

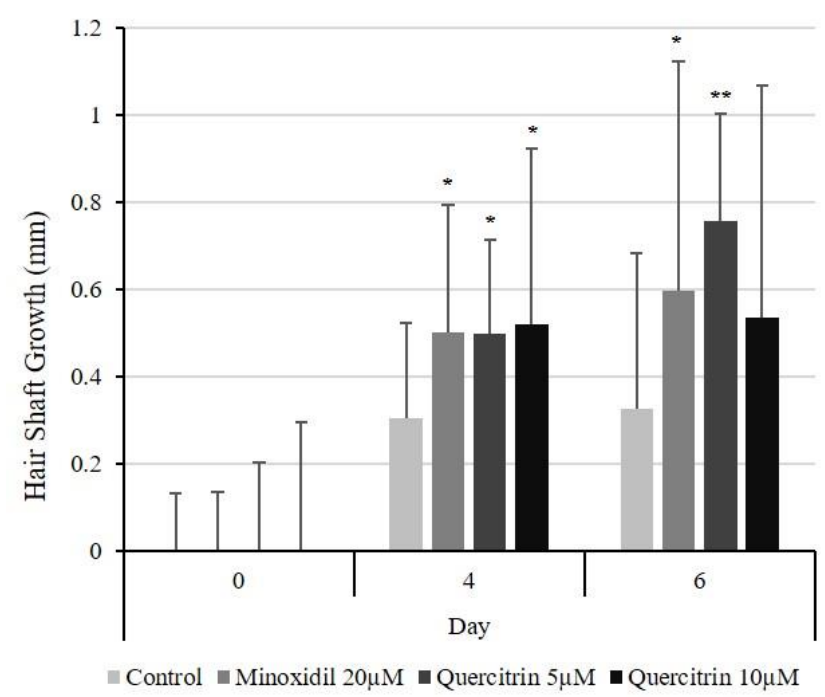

Figure 10. Effect of quercitrin on hair growth in human hair follicle organ culture. In order to evaluate the effect of quercitrin, the anagen human hair follicle were prepared and cultured for 6 days. Quercitrin was treated at concentrations of 5,10 $\mu \mathrm{M}$. (a) At day 4 and 6, the cultured hair follicles were photo-documented. (b) The hair shaft growth was analyzed. Minoxidil was used as a positive control. The data represent the means of sixteen follicles. Significantly different compared with N.T $(* p<0.05$, ** $\left.p<0.01,{ }^{* * *} p<0.001\right)$.

\section{Discussion}

The hair follicle is one of the most energy-consuming organs in humans so energy-genesis processes are highly activated in human hair follicle cells. In DPCs, mitochondria strongly contribute to energy generation [15], and the enhancement of mitochondrial function affects hair growth in vitro and in vivo [16]. Therefore, mitochondria and their energy production are regarded as important for promoting hair growth.

The mitochondrial energy generation is determined by mitochondrial membrane potential $(\Delta \Psi)$, which in turn is tightly conjugated with positive steady state of NAD/NADH coupling. Quercitrin treatment was found to increase not only NADH production, but also mitochondrial membrane potential (Figures $1 \mathrm{~b}$ and 2 ). In this context, our data strongly suggest that quercitrin could stimulate mitochondrial activities in human DPCs, which might be related to hair follicle morphogenesis [17].

The Bcl-2 family proteins were shown to manage cell senescence and administer cellular apoptosis [18]. Among the family members, Bcl2 in particular protects cells from stress challenges [19] and higher Bcl2 level prevents apoptosis [20]. In hair follicles, interestingly, the Bcl2 level is significantly higher in DPCs than other cell types [21]. In addition, high level of Bcl2 is essential for maintaining anagen and keeping DPCs function properly [22]. As mentioned above, Bcl2 level was increased and 
Bad level was decreased by quercitrin treatment (Figure 3). Our data demonstrate the possibility that quercitrin could maintain the hair follicle in anagen state.

In hair follicle, growth factors-such as bFGF, KGF, PDGF-AA, and VEGF-were reported to activate hair cycle and growth [8] and to be secreted by DPCs. Especially, KGF is one of the DPCs' signal that participates in instructing hair germ to proliferate and induce a new hair cycle [23]. In the present study, quercitrin was found to stimulate the production of these growth factors in cultured hDPCs (Figures 4-6). It could be predicted that the growth factors contributing to prolong the anagen and support hair growth could be supplied to hair bulb in higher level by quercitrin treatment.

As previously described, quercitrin activated Akt and Erk/CREB signaling in DPCs. The activation of CREB is strongly conjugated with mitochondrial activation, facilitating mitochondrial gene expression and cell survival through phosphor-CREB (Ser133) binding to CRE site in the mitochondrial D-loop DNA [24]. The cellular improvements are accompanied by corresponding expression of CREB-targeted genes, such as Bcl2 (cell survival) and PGC-1 $\alpha$ (mitochondrial biogenesis) [25]. These findings are consistent with our data that quercitrin stimulated Bcl-2 expression and mitochondrial function via activation of CREB.

Furthermore, many growth factors were reported to be regulated by MAPK/CREB signaling pathway. VEGF expression is regulated by PI3K/Akt-NF-KB cascade [26] or MAPK/ERK pathway [27]. The promoter region of the KGF gene has transcriptional regulatory element for activating transcription factor (ATF) family of CREB proteins [28], and CREB activation stimulate KGF gene expression in DPCs [29]. bFGF and PDGF-AA genes are also the target of early growth response gene-1 (EGR1) which is activated by Erk phosphorylation [30].

The receptor tyrosine kinase or related non-receptor tyrosine kinases, for example Src family proteins, are possibly involved in the activation of Akt and Erk pathways and the regulation of PI3K and MAPK pathways by Src family proteins are well-established [31]. As shown in Figure 9, several receptor tyrosine kinases and non-receptor tyrosine kinases were activated by quercitrin treatment. Among those, Src family proteins—such as Csk, FRK, Hck, and SRMS-are of special interest because they, as non-receptor tyrosine kinase, not only interact with the protein-tyrosine kinase receptors at cell membrane but also participate in numerous signaling pathways. Further studies for unveiling the role of Src family proteins in the activation of DPCs by quercitrin would be challenging.

In this report, we have found that quercitrin activated the energy metabolism and enhanced the production of growth factors including bFGF and KGF. We also found that Bcl2 and Ki67 levels were upregulated and Bad level was downregulated. It seems plausible that all these stimulations are related to the activation of Akt, Erk, and CREB signal transduction pathways (Figure 7). The Erk inhibitor U0126 but not Akt inhibitor API-2 was found to inhibit the quercitrin-induced expression of growth factors and Bcl2, strongly implying the involvement of Erk activation in its mechanism of action. The cell proliferation marker Ki67 was decreased by both Erk inhibitor U0126 and Akt inhibitor API-2. In addition, several receptor tyrosine kinases and their conjugated tyrosine kinases were also activated by quercitrin as an upstream of Akt and Erk/CREB signaling (Figure 9). Finally, quercitrin stimulated hair growth in a human hair follicle culture model, confirming our hypothesis.

We could not exclude, however, the possibility that the biological activities including hair growth promoting activity came from other constituents or unidentified ones of the HCE even though they took a little since other major components like chlorogenic acid and quercetin showed stimulatory activities in CCK-8 assay. Although quercitrin was our first choice for further investigation because of its abundance, investigating the roles of other major components of the HCE on hair growth might be interesting.

In conclusion, our data strongly suggest that quercitrin is the major active component of HCE and stimulates hair growth by enhancing the cellular energy metabolism and increasing the secretion of growth factors through the activation of MAPK/CREB signaling pathway, demonstrating the possible therapeutic potential of quercitrin for treating and/or preventing hair loss. 


\section{Materials and Methods}

\subsection{Dermal Papilla Cells Culture}

Human DPCs were purchased from Promocell (Heidelberg, Germany). DPCs were cultured in basal medium supplemented with $4 \%$ fetal calf serum, $0.4 \%$ bovine pituitary extract, $1 \mathrm{ng} / \mathrm{mL}$ basic fibroblast growth factor and $5 \mu \mathrm{g} / \mathrm{mL}$ insulin (Supplement Mix, Promocell). Cells were maintained in humidified incubator at $37^{\circ} \mathrm{C}, 5 \% \mathrm{CO}_{2}$. Before quercitrin (Sigma-Aldrich, MO, USA; Figure 1a) treatment, serum limitation was done by replacing the medium with fresh DMEM (Thermofisher scientific, Waltham, MA, USA) supplemented with 1\% FBS (Thermofisher scientific, Waltham, MA, USA) and $1 \mathrm{ng} / \mathrm{mL}$ bFGF (Merck, Darmstadt, Germany) and culturing for $24 \mathrm{~h}$ to minimize the effects of serum and growth supplements. Akt inhibitor API-2 and Erk inhibitor U0126 were purchased from Tocris Bioscience (Bristol, UK).

\subsection{Cell Viability Assay}

The effect of quercitrin on the viability of DPCs was examined using CCK-8 assay (Dojindo, MA, USA) and JC-1 mitochondrial membrane potential assay (Abcam, Cambridge, UK) kits following the manufacturer's protocols. For examining the cellular energy metabolism, NAD(P)H generation was measured by CCK-8 assay. The absorbance at $450 \mathrm{~nm}$ was read using micro-plate reader (BioTek, Winooski, VT, USA). The mitochondrial membrane potential was measured by JC-1 staining. Briefly, after quercitrin treated DPCs were stained with $1 \mu \mathrm{M}$ JC-1 solution, fluorescence intensities from JC-1 aggregate and monomer form were measured at $590 \mathrm{~nm}$ (535 nm excitation) and $530 \mathrm{~nm}(475 \mathrm{~nm}$ excitation), respectively, with Wallac Victor3 1420 (PerkinElmer, Waltham, MA, USA). Mitochondrial membrane potential $(\Delta \Psi)$ was visualized by taking fluorescence images with EVOSTM FL Auto2 Imaging System (Thermofisher scientific, Waltham, MA, USA).

\subsection{Quantitative Real-Time PCR}

Quercitrin was treated at concentrations of 1, 10, $100 \mathrm{nM}$ and $1 \mu \mathrm{M}$ for $24 \mathrm{~h}$, with non-treated cells served as control. Total RNA was extracted using Rneasy RNA extraction kit (Qiagen Inc., CA, Germantown, USA). cDNA synthesis was performed using cDNA synthesis kit (Phillkorea, Seoul, Korea) with ThermoCycler (R\&D systems, Minneapolis, MN, USA), according to the manufacturer's protocol. cDNA samples obtained from control and treated cells were subjected to real-time(RT) PCR analysis.

TaqMan probes for RT-PCR used in this study were as follows: GAPDH assay id 4352934E; Bcl2 assay id Hs00608023_m1; BAD assay id Hs00188930_m1; Bax assay id Hs00180269_m1; MKI67 assay id Hs04260396_g1; bFGF (FGF2) assay id Hs00266645_m1; KGF (FGF7) assay id Hs00940253_m1; IGFBP2 assay id Hs01040719_m1; TGFb1 assay id Hs00998133_m1; VEGFA assay id Hs00900055_m1; FGFR1 assay id Hs00241111_m1; PDGFRa assay id Hs01019589_m1; PDGFRb assay id Hs00998018_m1.

TaqMan One-Step RT-PCR Master Mix Reagent (Life Technologies, Carlsbad, CA, USA) was used. The PCR reactions were performed on ABI 7500 Real Time PCR system following the manufacturer's instruction. The resulting data were analyzed with ABI software (version).

\subsection{Western Blot Analysis}

DPCs $\left(1 \times 10^{6}\right.$ cells/dish) were seeded in $100 \mathrm{~mm}$ dishes and cultured for $24 \mathrm{~h}$. Quercitrin was treated at concentrations of 1, 10, and $100 \mathrm{nM}$ for appropriate time. The cells were then lysed and total cellular proteins were prepared. $50 \mu \mathrm{g}$ protein samples were analyzed by Western blotting with corresponding antibodies; Bcl2 (1:1000, Abcam, cambridge, UK), Akt (1:1000, Santa Cruz, CA, USA), Erk (p44/42) (1:1000, Cell Signaling Technology, Danvers, MA, USA), CREB-1 (Cell Signaling Technology, Danvers, MA, USA) GAPDH (1:2000, Santa Cruz, CA, USA), phospho (Ser473)-Akt (1:1000, Cell Signaling Technology, Danvers, MA, USA), phospho (Thr202/Tyr204)-Erk (p44/42) (1:1000, Cell Signaling Technology, Danvers, MA, USA), and phospho (Ser133)-CREB (1:1000, Cell Signaling 
Technology, Danvers, MA, USA). Western blot was analyzed by chemiluminescence detector iBright FL1000 (Invitrogen, Waltham, MA, USA).

\subsection{Protein Dot Blot Analysis for Growth Factors (Receptors) and Receptor Tyrosine Kinase Phosphorylation}

Human growth factor antibody array kit (Abcam, Cambridge, UK) and human receptor tyrosine kinase (RTK) phosphorylation antibody array kit (Abcam, Cambridge, UK) were used to elucidate the changes in growth factor profiles and signal transduction pathways in DPCs. Total of 41 human growth factors and 71 human RTK phosphorylation were analyzed. Cells were treated with $100 \mathrm{nM}$ and $1 \mu \mathrm{M}$ of quercitrin for appropriate time and then collected for growth factor and RTK phosphorylation analysis. Cells treated with vehicle medium were used as non-treated control. Conventional immunoblot process was performed following the manufacturer's protocol. The resulting blots were analyzed under identical condition using iBright FL1000 (Invitrogen, Waltham, MA, USA).

\section{6. bFGF and KGF ELISA}

DPCs were treated with various concentrations of quercitrin for $24 \mathrm{~h}$. The cells and culture medium were collected and the amounts of bFGF and KGF were measured using human bFGF and human KGF DuoSet ELISA (R\&D systems) kits, according to the manufacturer's instruction. Absorbance at $450 \mathrm{~nm}$ was measured using microplate reader (BioTek, Winooski, VT, USA). Background wavelength correction was done at $540 \mathrm{~nm}$.

\subsection{Human Hair Follicle Organ Culture}

Human scalp skin was obtained from nonbalding areas from patients undergoing hair transplant surgery with written consent and approval by the Institutional Review Board of Dankoon University Hospital (IRB no. DKUH. 2017-07-003). Human hair follicles were isolated by microdissection under the microscope. Anagen VI hair follicles were chosen for the study. Isolated hair follicles were maintained in William's E medium (Life Technologies, Carlsbad, CA, USA) supplemented with $10 \mu \mathrm{g} / \mathrm{mL}$ insulin (Sigma-Aldrich, St. Louis, MO, USA), $10 \mathrm{ng} / \mathrm{mL}$ hydrocortisone (Sigma-Aldrich, St. Louis, MO, USA), $2 \mathrm{mM}$ L-glutamine (Life Technologies, Carlsbad, CA, USA), and $10 \mathrm{U} / \mathrm{mL}$ penicillin (Life Technologies, Carlsbad, CA, USA), $100 \mathrm{ug} / \mathrm{mL}$ streptomycin (Life Technologies, Carlsbad, CA, USA), and $25 \mathrm{ug} / \mathrm{mL}$ amphotericin B (Life Technologies, Carlsbad, CA, USA). All cultures were incubated at $37^{\circ} \mathrm{C}$ in an atmosphere of $5 \% \mathrm{CO}_{2}$ and $95 \%$ air.

\subsection{Statistical Analysis}

All experimental data were presented as the mean \pm standard deviation (S.D.) of at least three independent experiments. Experimental results were analyzed using the SigmaPlot 8.0 (Systat Software Inc., Chicago, IL, USA). The statistical significance of difference was determined by Student's $t$-test. The value of $p<0.05$ considered statistically significant.

Author Contributions: Conceptualization, J.K.; Data curation, J.K., S.R.K., Y.-H.C., and J.y.S.; Formal analysis, J.K. S.R.K., and B.C.P.; Investigation, N.-G.K. and S.L.; Methodology, J.K.; Project administration, S.L.; Supervision, S.L.; Validation, B.C.P.; Visualization, J.K. Writing—original draft, J.K.; Writing—review and editing, C.D.K., N.-G.K., and S.L. All authors have read and agreed to the published version of the manuscript.

Funding: This research received no external funding.

Conflicts of Interest: The authors declare no conflict of interest.

\section{References}

1. Monk, B. Hair and its Disorders: Biology, Pathology and Management. J. R. Soc. Med. 2001, $94,549$. [CrossRef]

2. Lolli, F.; Pallotti, F.; Rossi, A.; Fortuna, M.C.; Caro, G.; Lenzi, A.; Sansone, A.; Lombardo, F. Androgenetic alopecia: A review. Endocrine 2017, 57, 9-17. [CrossRef] [PubMed] 
3. Han, S.-H.; Byun, J.-W.; Lee, W.-S.; Kang, H.; Kye, Y.-C.; Kim, K.-H.; Kim, D.-W.; Kim, M.-B.; Kim, S.-J.; Kim, H.-O. Quality of life assessment in male patients with androgenetic alopecia: Result of a prospective, multicenter study. Ann. Dermatol. 2012, 24, 311-318. [CrossRef] [PubMed]

4. DeVillez, R.L. The Therapeutic Use of Topical Minoxidil. Dermatol. Clin. 1990, 8, 367-375. [CrossRef]

5. Paus, R.; Muller-Rover, S.; Botchkarev, V.A. Chronobiology of the hair follicle: Hunting the "hair cycle clock". J. Investig. Derm. Symp. Proc. 1999, 4, 338-345. [CrossRef]

6. Rutberg, S.E.; Kolpak, M.L.; Gourley, J.A.; Tan, G.; Henry, J.P.; Shander, D. Differences in Expression of Specific Biomarkers Distinguish Human Beard from Scalp Dermal Papilla Cells. J. Investig. Dermatol. 2006, 126, 2583-2595. [CrossRef]

7. Gentile, P.; Cole, J.P.; Cole, M.A.; Garcovich, S.; Bielli, A.; Scioli, M.G.; Orlandi, A.; Insalaco, C.; Cervelli, V. Evaluation of Not-Activated and Activated PRP in Hair Loss Treatment: Role of Growth Factor and Cytokine Concentrations Obtained by Different Collection Systems. Int. J. Mol. Sci. 2017, 18, 408. [CrossRef]

8. Gentile, P.; Garcovich, S. Advances in Regenerative Stem Cell Therapy in Androgenic Alopecia and Hair Loss: Wnt pathway, Growth-Factor, and Mesenchymal Stem Cell Signaling Impact Analysis on Cell Growth and Hair Follicle Development. Cells 2019, 8, 466. [CrossRef]

9. Kim, J.; Shin, J.Y.; Choi, Y.H.; Jang, M.; Nam, Y.J.; Lee, S.Y.; Jeon, J.; Jin, M.H.; Lee, S. Hair Growth Promoting Effect of Hottuynia cordata Extract in Cultured Human Hair Follicle Dermal Papilla Cells. Biol. Pharm. Bull. 2019, 42, 1665-1673. [CrossRef]

10. Comalada, M.; Camuesco, D.; Sierra, S.; Ballester, I.; Xaus, J.; Galvez, J.; Zarzuelo, A. In vivo quercitrin anti-inflammatory effect involves release of quercetin, which inhibits inflammation through down-regulation of the NF-kappaB pathway. Eur. J. Immunol. 2005, 35, 584-592. [CrossRef]

11. Yin, Y.; Li, W.; Son, Y.O.; Sun, L.; Lu, J.; Kim, D.; Wang, X.; Yao, H.; Wang, L.; Pratheeshkumar, P.; et al. Quercitrin protects skin from UVB-induced oxidative damage. Toxicol. Appl. Pharmacol. 2013, 269, 89-99. [CrossRef] [PubMed]

12. Li, W.; Man, X.Y.; Li, C.M.; Chen, J.Q.; Zhou, J.; Cai, S.Q.; Lu, Z.F.; Zheng, M. VEGF induces proliferation of human hair follicle dermal papilla cells through VEGFR-2-mediated activation of ERK. Exp. Cell Res. 2012, 318, 1633-1640. [CrossRef] [PubMed]

13. Xing, J.; Kornhauser, J.M.; Xia, Z.; Thiele, E.A.; Greenberg, M.E. Nerve growth factor activates extracellular signal-regulated kinase and p38 mitogen-activated protein kinase pathways to stimulate CREB serine 133 phosphorylation. Mol. Cell. Biol. 1998, 18, 1946-1955. [CrossRef] [PubMed]

14. Mendoza, M.C.; Er, E.E.; Blenis, J. The Ras-ERK and PI3K-mTOR pathways: Cross-talk and compensation. Trends Biochem. Sci. 2011, 36, 320-328. [CrossRef]

15. Lemasters, J.J.; Ramshesh, V.K.; Lovelace, G.L.; Lim, J.; Wright, G.D.; Harland, D.; Dawson, T.L., Jr. Compartmentation of Mitochondrial and Oxidative Metabolism in Growing Hair Follicles: A Ring of Fire. J. Investig. Derm. 2017, 137, 1434-1444. [CrossRef]

16. Foitzik, K.; Hoting, E.; Heinrich, U.; Tronnier, H.; Paus, R. Indications that topical L-carnitin-L-tartrate promotes human hair growth in vivo. J. Derm. Sci 2007, 48, 141-144. [CrossRef]

17. Vidali, S.; Knuever, J.; Lerchner, J.; Giesen, M.; Biro, T.; Klinger, M.; Kofler, B.; Funk, W.; Poeggeler, B.; Paus, R. Hypothalamic-pituitary-thyroid axis hormones stimulate mitochondrial function and biogenesis in human hair follicles. J. Investig. Derm. 2014, 134, 33-42. [CrossRef]

18. Yosef, R.; Pilpel, N.; Tokarsky-Amiel, R.; Biran, A.; Ovadya, Y.; Cohen, S.; Vadai, E.; Dassa, L.; Shahar, E.; Condiotti, R.; et al. Directed elimination of senescent cells by inhibition of BCL-W and BCL-XL. Nat. Commun. 2016, 7, 11190. [CrossRef]

19. Deng, X.; Kornblau, S.M.; Ruvolo, P.P.; May, W.S., Jr. Regulation of Bcl2 phosphorylation and potential significance for leukemic cell chemoresistance. J. Natl. Cancer Inst. Monogr. 2000, 28, 30-37. [CrossRef]

20. Song, H.; Han, I.Y.; Kim, Y.; Kim, Y.H.; Choi, I.W.; Seo, S.K.; Jung, S.Y.; Park, S.; Kang, M.S. The NADPH oxidase inhibitor DPI can abolish hypoxia-induced apoptosis of human kidney proximal tubular epithelial cells through Bcl2 up-regulation via ERK activation without ROS reduction. Life Sci. 2015, 126, 69-75. [CrossRef]

21. Stenn, K.S.; Lawrence, L.; Veis, D.; Korsmeyer, S.; Seiberg, M. Expression of the bcl-2 protooncogene in the cycling adult mouse hair follicle. J. Investig. Derm. 1994, 103, 107-111. [CrossRef] [PubMed]

22. Botchkareva, N.V.; Ahluwalia, G.; Shander, D. Apoptosis in the hair follicle. J. Investig. Derm. 2006, 126, 258-264. [CrossRef] [PubMed] 
23. Greco, V.; Chen, T.; Rendl, M.; Schober, M.; Pasolli, H.A.; Stokes, N.; Dela Cruz-Racelis, J.; Fuchs, E. A two-step mechanism for stem cell activation during hair regeneration. Cell Stem Cell 2009, 4, 155-169. [CrossRef] [PubMed]

24. Ryu, H.; Lee, J.; Impey, S.; Ratan, R.R.; Ferrante, R.J. Antioxidants modulate mitochondrial PKA and increase CREB binding to D-loop DNA of the mitochondrial genome in neurons. Proc. Natl. Acad. Sci. USA 2005, 102, 13915-13920. [CrossRef]

25. Nichols, M.; Zhang, J.; Polster, B.M.; Elustondo, P.A.; Thirumaran, A.; Pavlov, E.V.; Robertson, G.S. Synergistic neuroprotection by epicatechin and quercetin: Activation of convergent mitochondrial signaling pathways. Neuroscience 2015, 308, 75-94. [CrossRef]

26. Tong, Q.; Zheng, L.; Lin, L.; Li, B.; Wang, D.; Huang, C.; Li, D. VEGF is upregulated by hypoxia-induced mitogenic factor via the PI-3K/Akt-NF-kappaB signaling pathway. Respir. Res. 2006, 7, 37. [CrossRef]

27. Moon, E.J.; Sonveaux, P.; Porporato, P.E.; Danhier, P.; Gallez, B.; Batinic-Haberle, I.; Nien, Y.C.; Schroeder, T.; Dewhirst, M.W. NADPH oxidase-mediated reactive oxygen species production activates hypoxia-inducible factor-1 (HIF-1) via the ERK pathway after hyperthermia treatment. Proc. Natl. Acad. Sci. USA 2010, 107, 20477-20482. [CrossRef]

28. Zhou, J.; Finch, P.W. Identification of a novel transcriptional regulatory element within the promoter region of the keratinocyte growth factor gene that mediates inducibility to cyclic AMP. Biochim. Biophys. Acta 1999, 1446, 71-81. [CrossRef]

29. Iino, M.; Ehama, R.; Nakazawa, Y.; Iwabuchi, T.; Ogo, M.; Tajima, M.; Arase, S. Adenosine stimulates fibroblast growth factor-7 gene expression via adenosine A2b receptor signaling in dermal papilla cells. J. Investig. Derm. 2007, 127, 1318-1325. [CrossRef]

30. Ma, Y.; Han, C.C.; Li, Y.; Wang, Y.; Wei, W. Insulin-like growth factor-binding protein-3 inhibits IGF-1-induced proliferation of human hepatocellular carcinoma cells by controlling bFGF and PDGF autocrine/paracrine loops. Biochem Biophys Res. Commun 2016, 478, 964-969. [CrossRef]

31. Roskoski, R., Jr. Src protein-tyrosine kinase structure, mechanism, and small molecule inhibitors. Pharmacol. Res. 2015, 94, 9-25. [CrossRef] [PubMed]

Sample Availability: All samples in these studies are available.

(C) 2020 by the authors. Licensee MDPI, Basel, Switzerland. This article is an open access article distributed under the terms and conditions of the Creative Commons Attribution (CC BY) license (http://creativecommons.org/licenses/by/4.0/). 